\title{
On maximal immediate extensions of valued division algebras
}

\author{
Erwin Schörner \\ (Communicated by Rüdiger Göbel)
}

\begin{abstract}
We show an extension theorem for strictly contracting bilinear mappings into a spherically complete valued vector space and we apply this result to prove that every maximal valued division algebra having the same characteristic as its residue division algebra is spherically complete.
\end{abstract}

2000 Mathematics Subject Classification: 12K10, 17A80, 46S10.

In his classical paper [5], Kaplansky proved that a valued field $\left(K, v, \Gamma_{0}\right)$ is maximal if and only if any pseudoconvergent sequence has a pseudolimit in $K$; moreover, under the "Hypothesis A", $\left(K, v, \Gamma_{0}\right)$ is isomorphic to a Hahn field of formal power series with a factor system. The equivalence of maximality and pseudocompleteness can also be shown for valued abelian groups (see [3], [9] and [11]) and certain classes of valued modules (see [6]). It is still an open question for valued skewfields; in this context, Brungs and Törner gave an example of a maximal right chain ring which is not pseudocomplete (see [2]).

The purpose of this paper is to state the positive result for a valued division algebra in the sense of Zelinsky [16] having the same characteristic as its residue division algebra. This is a generalization of [15, Satz 5] where we give criteria for the embeddability of a valued division algebra into an appropriate Hahn division algebra. Here, we also rely on these Hahn division algebras of formal power series constructed and studied in [12] and [13], but we have to modify their multiplication applying an extension theorem for strictly contracting mappings into a spherically complete valued vector space.

Omitting the algebraic structure of the objects mentioned above, we obtain an ultrametric space (with a totally ordered value set). The theory of ultrametric spaces (even with partially ordered value set) was developed by Prieß-Crampe and Ribenboim in their papers [8], [9] and [10]; for the convenience of the reader we will recall the main results of this theory we are going to make use of in the sequel.

Let $X$ be a set, and let $(\Gamma, \leq)$ be a (totally) ordered set and $\Gamma_{0}=\Gamma \cup\{0\}$ with $0<\gamma$ for all $\gamma \in \Gamma$. A mapping $d: X \times X \rightarrow \Gamma_{0}$ is called an ultrametric distance, if the following conditions are satisfied for all $x, y$ and $z \in X$ : 
- $d(x, y)=0 \Leftrightarrow x=y$.

- $d(x, y)=d(y, x)$.

- $d(x, z) \leq \operatorname{Max}\{d(x, y), d(y, z)\}$.

In this situation, $\left(X, d, \Gamma_{0}\right)$ is called an ultrametric space. For $x, y, z \in X$ with $d(x, y) \neq$ $d(y, z)$ we even have $d(x, z)=\operatorname{Max}\{d(x, y), d(y, z)\}$.

An equivalence relation $\sigma$ on $X$ is called $d$-compatible, if for all $x, x^{\prime}, y, y^{\prime} \in X$ with $x \sigma y$ and $d\left(x^{\prime}, y^{\prime}\right) \leq d(x, y)$ we also have $x^{\prime} \sigma y^{\prime}$. The set $\equiv(X)$ of all $d$-compatible equivalence relations on $X$ is a complete totally ordered set with respect to $\subseteq$. The most important examples are $\equiv_{\gamma}$ and $\equiv_{\gamma}^{-}$for a $\gamma \in \Gamma$ with $x \equiv_{\gamma} y \Leftrightarrow d(x, y) \leq \gamma$ and $x \equiv \equiv_{\gamma}^{-} y \Leftrightarrow d(x, y)<\gamma$ for all $x, y \in X$, respectively. The equivalence classes of $\equiv_{\gamma}$ and $\equiv_{\gamma}^{-}$are precisely the balls $X^{\gamma}(x)=\{y \in X \mid d(x, y) \leq \gamma\}$ and $X_{\gamma}(x)=$ $\{y \in X \mid d(x, y)<\gamma\}$ with centre $x$ and radius $\gamma$, respectively. Any set of pairwise nondisjoint balls is a chain with respect to $\subseteq$.

By [7], the following completeness properties are equivalent:

- $\left(X, d, \Gamma_{0}\right)$ is spherically complete: any chain of balls $X^{\gamma}(x)$ with $x \in X$ and $\gamma \in \Gamma$ has a non-empty intersection.

- $\left(X, d, \Gamma_{0}\right)$ is pseudocomplete: any pseudoconvergent sequence has a pseudolimit in $X$.

- $\left(X, d, \Gamma_{0}\right)$ satisfies the ultrametric Banach's Fixed Point Theorem: any strictly contracting mapping $f: X \rightarrow X$, i.e., $d(f(x), f(y))<d(x, y)$ holds for all $x, y \in X$ with $x \neq y$, has a fixed point in $X$.

Analyzing the proof of $[7$, Satz 1$]$, we realize that in a spherically complete ultrametric space any chain of balls has a non-empty intersection.

A subset $U$ of $X$ endowed with the restriction $d=\left.d\right|_{U \times U}$ of $d$ to $U$ is again an ultrametric space. The extension $\left(U, d, \Gamma_{0}\right) \prec\left(X, d, \Gamma_{0}\right)$ is said to be immediate, if $d(U \times U)=d(X \times X)$ holds and if for all $u \in U$ and $x \in X$ with $u \neq x$ there exists $u^{\prime} \in U$ with $d\left(u^{\prime}, x\right)<d(u, x)$. An ultrametric space without any proper immediate extension is called maximal. By [10, Theorem 7.9] and [11, Theorem 2.3], an ultrametric space is spherically complete if and only if it is maximal.

The most important examples of spherically complete ultrametric spaces are the Hahn spaces of formal power series; in this paper, we only consider a special case. Let $\Gamma_{0}$ be a totally ordered set as above, and let $M$ be a set with at least two elements and $0 \in M$. The Hahn space $\mathbf{H}=\left(\mathbf{H}, d_{\mathbf{H}}, \Gamma_{0}\right)$ consists of all mappings $\mathbf{f}: \Gamma \rightarrow M$ with dually well-ordered support $\operatorname{supp}(\mathbf{f})=\{\gamma \in \Gamma \mid \mathbf{f}(\gamma) \neq 0\}$, i.e., $\operatorname{supp}(\mathbf{f})$ is well-ordered with respect to the opposite order, and carries the ultrametric distance

$$
d_{\mathbf{H}}(\mathbf{f}, \mathbf{g})=\left\{\begin{array}{ll}
\operatorname{Max}\{\gamma \in \Gamma \mid \mathbf{f}(\gamma) \neq \mathbf{g}(\gamma)\}, & \text { if } \mathbf{f} \neq \mathbf{g} \\
0, & \text { if } \mathbf{f}=\mathbf{g}
\end{array} .\right.
$$

Usually, the formal power series $\mathbf{f} \in \mathbf{H}$ is symbolized by $\sum_{\gamma \in \Gamma} \mathbf{f}(\gamma) t^{\gamma}$ using the indeterminate $t$; thus, $m t^{\gamma}$ represents the element of $\mathbf{H}$ with 


$$
\left(m t^{\gamma}\right)\left(\gamma^{\prime}\right)= \begin{cases}m, & \text { if } \gamma^{\prime}=\gamma \\ 0, & \text { if } \gamma^{\prime} \neq \gamma\end{cases}
$$

for $m \in M$ and $\gamma \in \Gamma$.

We use the same definition of a valued group and a valued field as [9]; for the convenience of the reader, we recall the notion of a valued division algebra. We consider a division algebra $(N,+, \cdot)$, i.e.,

- $(N,+)$ is an abelian group with neutral element 0 ,

- $\left(N^{*}, \cdot\right)$ with $N^{*}=N \backslash\{0\}$ is a loop with neutral element 1 ,

- $a \cdot(b+c)=a \cdot b+a \cdot c$ and $(a+b) \cdot c=a \cdot c+b \cdot c$ hold for all $a, b, c \in N$.

Hence, a division algebra with an associative multiplication is a skewfield. Let $\Gamma$ be endowed with a multiplication $\cdot$ such that $(\Gamma, \cdot, \varepsilon, \leq)$ becomes a totally ordered loop with neutral element $\varepsilon$; we extend the multiplication to $\Gamma_{0} \times \Gamma_{0} \rightarrow \Gamma_{0}$ by putting $\gamma \cdot 0=0$ and $0 \cdot \gamma=0$ for all $\gamma \in \Gamma_{0}$. A mapping $v: N \rightarrow \Gamma_{0}$ is called a valuation, if the following conditions are satisfied for all $x$ and $y \in N$ :

- $v(x)=0 \Leftrightarrow x=0$.

- $v(x \cdot y)=v(x) \cdot v(y)$.

- $v(x+y) \leq \operatorname{Max}\{v(x), v(y)\}$.

In this situation, $\left(N, v, \Gamma_{0}\right)$ is called a valued division algebra. This can be regarded as a special case of the concept of a uniformly valued ternary field developed by Kalhoff in [4]; we should mention that $\Gamma$ carries the dual order $\leq_{d}$ in [16], i.e., for all $\gamma, \gamma^{\prime} \in \Gamma$ we have $\gamma \leq_{d} \gamma^{\prime}$ if and only if $\gamma^{\prime} \leq \gamma$ holds.

For all these algebraic structures, the valuation $v$ induces an ultrametric distance $d_{v}$ by $d_{v}(x, y)=v(x-y)$ for all $x$ and $y$.

In the following, we transfer the general construction of a Hahn ternary field of formal power series given in [12] to our special situation. Therefore, we consider a division algebra $K$ and a totally ordered loop $(\Gamma, \cdot, \varepsilon, \leq)$. For all $\alpha, \beta \in \Gamma$ let $\mu_{\alpha, \beta}: K \times K \rightarrow K$ be a biadditive mapping satisfying the following conditions:

- For all $m, b \in K$ with $m \neq 0$ there is a unique $x \in K$ with $\mu_{\alpha, \beta}(m, x)=b$.

- For all $x, b \in K$ with $x \neq 0$ there is $m \in K$ with $\mu_{\alpha, \beta}(m, x)=b$.

- $\mu_{\varepsilon, \varepsilon}(m, x)=m \cdot x, \mu_{\alpha, \varepsilon}(m, 1)=m$ and $\mu_{\varepsilon, \beta}(1, x)=x$ hold for all $m, x \in K$.

In this case, the family $\left(\mu_{\alpha, \beta}\right)_{\alpha, \beta \in \Gamma}$ is called a factor system with respect to $K$ and $\Gamma$. We define addition and multiplication on the set $\mathbf{H}$ by putting

$$
(\mathbf{f}+\mathbf{g})(\gamma)=\mathbf{f}(\gamma)+\mathbf{g}(\gamma) \quad \text { and } \quad(\mathbf{f} \cdot \mathbf{g})(\gamma)=\sum_{\alpha \cdot \beta=\gamma} \mu_{\alpha, \beta}(\mathbf{f}(\alpha), \mathbf{g}(\beta))
$$

for all $\mathbf{f}, \mathbf{g} \in \mathbf{H}$ and $\gamma \in \Gamma$. By [12, Satz 6] and [13, Satz 3 and Satz 4], $(\mathbf{H},+, \cdot)$ is a division algebra with $\mathbf{0}=0 t^{\varepsilon}$ and $\mathbf{1}=1 t^{\varepsilon}$; moreover, $\left(\mathbf{H}, v_{\mathbf{H}}, \Gamma_{0}\right)$ is a valued division algebra with $v_{\mathbf{H}}(\mathbf{f})=d_{\mathbf{H}}(\mathbf{f}, \mathbf{0})$ for all $\mathbf{f} \in \mathbf{H}$. 
First, we want to state and prove the extension theorem for bilinear mappings already mentioned above. Therefore, we need a more general concept of a valued vector space than that given in [17]. We consider a valued field $\left(K, v, \Gamma_{0}\right)$ and a vector space $V$ over $K$ endowed with a group valuation $\|\cdot\|: V \rightarrow \Gamma_{0}^{V}$. Let $\cdot: \Gamma \times \Gamma^{V} \rightarrow \Gamma^{V}$ be an operation of the group $\Gamma$ on the set $\Gamma^{V}$ such that

$$
\gamma^{\prime}<\gamma^{\prime \prime} \Rightarrow \gamma^{\prime} \cdot \gamma_{V}<\gamma^{\prime \prime} \cdot \gamma_{V} \quad \text { and } \quad \gamma_{V}^{\prime}<\gamma_{V}^{\prime \prime} \Rightarrow \gamma \cdot \gamma_{V}^{\prime}<\gamma \cdot \gamma_{V}^{\prime \prime}
$$

hold for all $\gamma, \gamma^{\prime}, \gamma^{\prime \prime} \in \Gamma$ and $\gamma_{V}, \gamma_{V}^{\prime}, \gamma_{V}^{\prime \prime} \in \Gamma^{V}$; we extend this operation to $\Gamma_{0} \times \Gamma_{0}^{V} \rightarrow \Gamma_{0}^{V}$ by putting $\gamma \cdot 0=0$ for all $\gamma \in \Gamma_{0}$ and $0 \cdot \gamma_{V}=0$ for all $\gamma_{V} \in \Gamma_{0}^{V}$. In this situation, we call $(V,\|\cdot\|)$ a valued vector space over $(K, v)$, if

$$
\|\lambda x\|=v(\lambda) \cdot\|x\|
$$

is satisfied for all $\lambda \in K$ and $x \in V$. We wish to remark that further assumptions are necessary to ensure that $V$ endowed with the topology $\mathfrak{I}_{\|\cdot\|}$ induced by the valuation $\|\cdot\|$ is a topological vector space over the topological field $\left(K, \mathfrak{I}_{v}\right)$, where $\mathfrak{I}_{v}$ is given by the valuation $v$. For example, it is sufficient to ask that $\Gamma \cdot \gamma_{V}$ is a coinitial subset of $\Gamma^{V}$ for all $\gamma_{V} \in \Gamma^{V}$.

Let $\left(X,\|\cdot\|_{X}\right),\left(Y,\|\cdot\|_{Y}\right)$ and $\left(Z,\|\cdot\|_{Z}\right)$ be valued vector spaces over the valued field $\left(K, v, \Gamma_{0}\right)$. A mapping $\circ: \Gamma^{X} \times \Gamma^{Y} \rightarrow \Gamma^{Z}$ is called value-multiplication, if the properties

$$
\gamma_{X}^{\prime}<\gamma_{X}^{\prime \prime} \Rightarrow \gamma_{X}^{\prime} \circ \gamma_{Y}<\gamma_{X}^{\prime \prime} \circ \gamma_{Y} \quad \text { and } \quad \gamma_{Y}^{\prime}<\gamma_{Y}^{\prime \prime} \Rightarrow \gamma_{X} \circ \gamma_{Y}^{\prime}<\gamma_{X} \circ \gamma_{Y}^{\prime \prime}
$$

and

$$
\gamma \cdot\left(\gamma_{X} \circ \gamma_{Y}\right)=\left(\gamma \cdot \gamma_{X}\right) \circ \gamma_{Y}=\gamma_{X} \circ\left(\gamma \cdot \gamma_{Y}\right)
$$

hold for all $\gamma \in \Gamma, \gamma_{X}, \gamma_{X}^{\prime}, \gamma_{X}^{\prime \prime} \in \Gamma^{X}$ and $\gamma_{Y}, \gamma_{Y}^{\prime}, \gamma_{Y}^{\prime \prime} \in \Gamma^{Y}$; again, we extend this valuemultiplication to $\Gamma_{0}^{X} \times \Gamma_{0}^{Y} \rightarrow \Gamma_{0}^{Z}$ by defining $\gamma_{X} \circ 0=0$ for all $\gamma_{X} \in \Gamma_{0}^{X}$ and $0 \circ \gamma_{Y}=0$ for all $\gamma_{Y} \in \Gamma_{0}^{Y}$. Then, a bilinear mapping $f: X \times Y \rightarrow Z$ is called strictly contracting with respect to the value-multiplication $\circ$, if

$$
\|f(x, y)\|_{Z}<\|x\|_{X} \circ\|y\|_{Y}
$$

is satisfied for all $0 \neq x \in X$ and $0 \neq y \in Y$.

Theorem 1. Let $\left(X,\|\cdot\|_{X}, \Gamma_{0}^{X}\right),\left(Y,\|\cdot\|_{Y}, \Gamma_{0}^{Y}\right)$ and $\left(Z,\|\cdot\|_{Z}, \Gamma_{0}^{Z}\right)$ be valued vector spaces over the valued field $\left(K, v, \Gamma_{0}\right)$ and $\circ: \Gamma^{X} \times \Gamma^{Y} \rightarrow \Gamma^{Z}$ a value-multiplication. Let $U$ be a linear subspace of $X$ and $f: U \times Y \rightarrow Z$ a bilinear mapping which is strictly contracting with respect to $\circ$; let $V$ be a linear subspace of $Y$ with $f(x, y)=0$ for all $x \in U$ and $y \in V$. If $\left(Z, d_{\|\cdot\|_{Z}}, \Gamma_{0}^{Z}\right)$ is spherically complete, then $f$ extends to a bilinear mapping $F: X \times Y \rightarrow Z$ which is strictly contracting with respect to $\circ$ and satisfies $F(x, y)=0$ for all $x \in X$ and $y \in V$. 
Proof. Let $\mathfrak{M}$ be the set of all pairs $\left(U^{\prime}, f^{\prime}\right)$ where $U^{\prime}$ is a linear subspace of $X$ containing $U$ and $f^{\prime}: U^{\prime} \times Y \rightarrow Z$ is a bilinear extension of $f$ which is strictly contracting with respect to $\circ$ and satisfies $f^{\prime}(x, y)=0$ for all $x \in U^{\prime}$ and $y \in V$. Since $(U, f) \in \mathfrak{M}$, the set $\mathfrak{M}$ is non-empty. Moreover, $\mathfrak{M}$ is inductively ordered by

$$
\left(U^{\prime}, f^{\prime}\right) \leq\left(U^{\prime \prime}, f^{\prime \prime}\right) \Leftrightarrow U^{\prime} \subseteq U^{\prime \prime} \text { and }\left.f^{\prime \prime}\right|_{U^{\prime} \times Y}=f^{\prime}
$$

and therefore contains a maximal element $\left(U_{0}, f_{0}\right)$ by Zorn's lemma. In the following, we show that the assumption $U_{0} \subsetneq X$ yields a contradiction.

Let $s \in X \backslash U_{0}$ and $U_{1}=U_{0} \oplus K s \subseteq X$. For all $u \in U_{0}$ we define $\pi_{u}=\|s-u\|_{X} \in \Gamma^{X}$.

Let $\mathfrak{N}$ be the set of all pairs $\left(V^{\prime}, g^{\prime}\right)$ where $V^{\prime}$ is a linear subspace of $Y$ containing $V$ and $g^{\prime}: V^{\prime} \rightarrow Z$ is a linear mapping with

$$
g^{\prime}(y) \in Z_{\pi_{u} \circ\|y\|_{Y}}\left(f_{0}(u, y)\right) \quad \text { for all } u \in U_{0} \quad \text { and } \quad 0 \neq y \in V^{\prime}
$$

and $g^{\prime}(y)=0$ for all $y \in V$. By $(V, 0) \in \mathfrak{N}$, the set $\mathfrak{N}$ is non-empty. Moreover, $\mathfrak{N}$ is inductively ordered by

$$
\left(V^{\prime}, g^{\prime}\right) \leq\left(V^{\prime \prime}, g^{\prime \prime}\right) \Leftrightarrow V^{\prime} \subseteq V^{\prime \prime} \quad \text { and }\left.\quad g^{\prime \prime}\right|_{V^{\prime}}=g^{\prime}
$$

and therefore contains a maximal element $\left(V_{0}, g_{0}\right)$ by Zorn's lemma. We now show that the assumption $V_{0} \subsetneq Y$ is absurd.

Let $t \in Y \backslash V_{0}$ and $V_{1}=V_{0} \oplus K t \subseteq Y$. For $u \in U_{0}$ and $y \in V_{0}$ we define the ball

$$
B_{u, y}=Z_{\pi_{u} \circ\|y+t\|_{Y}}\left(f_{0}(u, y+t)-g_{0}(y)\right)
$$

and we show that the intersection of any two of these balls is non-empty. To this end, let $u, u^{\prime} \in U_{0}$ with $\pi_{u} \leq \pi_{u^{\prime}}$ and $y, y^{\prime} \in V_{0}$. In the case $u \neq u^{\prime}$ and $y=y^{\prime}$ we have

$$
\begin{aligned}
& \left\|\left(f_{0}(u, y+t)-g_{0}(y)\right)-\left(f_{0}\left(u^{\prime}, y+t\right)-g_{0}(y)\right)\right\|_{Z} \\
& \quad=\left\|f_{0}\left(u-u^{\prime}, y+t\right)\right\|_{Z}<\left\|u-u^{\prime}\right\|_{X} \circ\|y+t\|_{Y} \leq \pi_{u^{\prime}} \circ\|y+t\|_{Y}
\end{aligned}
$$

and $B_{u, y} \subseteq B_{u^{\prime}, y}$. In the case $u=u^{\prime}$ and $y \neq y^{\prime}$ we have

$$
\begin{aligned}
& \left\|\left(f_{0}(u, y+t)-g_{0}(y)\right)-\left(f_{0}\left(u, y^{\prime}+t\right)-g_{0}\left(y^{\prime}\right)\right)\right\|_{Z} \\
& =\left\|f_{0}\left(u, y-y^{\prime}\right)-g_{0}\left(y-y^{\prime}\right)\right\|_{Z}<\pi_{u} \circ\left\|y-y^{\prime}\right\|_{Y} \\
& \quad \leq \operatorname{Max}\left\{\pi_{u} \circ\|y+t\|_{Y}, \pi_{u} \circ\left\|y^{\prime}+t\right\|_{Y}\right\}
\end{aligned}
$$

and $B_{u, y^{\prime}} \subseteq B_{u, y}$ or $B_{u, y} \subseteq B_{u, y^{\prime}}$. Finally, in the case $u \neq u^{\prime}$ and $y \neq y^{\prime}$ we obtain 


$$
\begin{aligned}
& \left\|\left(f_{0}(u, y+t)-g_{0}(y)\right)-\left(f_{0}\left(u^{\prime}, y^{\prime}+t\right)-g_{0}\left(y^{\prime}\right)\right)\right\|_{Z} \\
& \quad=\left\|\left(f_{0}\left(u, y-y^{\prime}\right)-g_{0}\left(y-y^{\prime}\right)\right)+f_{0}\left(u-u^{\prime}, y^{\prime}+t\right)\right\|_{Z} \\
& \quad \leq \operatorname{Max}\left\{\left\|\left(f_{0}\left(u, y-y^{\prime}\right)-g_{0}\left(y-y^{\prime}\right)\right)\right\|_{Z},\left\|f_{0}\left(u-u^{\prime}, y^{\prime}+t\right)\right\|_{Z}\right\} \\
& \quad<\operatorname{Max}\left\{\pi_{u} \circ\left\|y-y^{\prime}\right\|_{Y},\left\|u-u^{\prime}\right\|_{X} \circ\left\|y^{\prime}+t\right\|_{Y}\right\} \\
& \quad \leq \operatorname{Max}\left\{\pi_{u} \circ\|y+t\|_{Y}, \pi_{u^{\prime}} \circ\left\|y^{\prime}+t\right\|_{Y}\right\}
\end{aligned}
$$

and $B_{u^{\prime}, y^{\prime}} \subseteq B_{u, y}$ or $B_{u, y} \subseteq B_{u^{\prime}, y^{\prime}}$. Since $\left(Z,\|\cdot\|_{Z}, \Gamma_{0}^{Z}\right)$ is spherically complete, there exists

$$
g_{1}(t) \in \bigcap_{u \in U_{0}} \bigcap_{y \in V_{0}} B_{u, y} .
$$

Therefore, $g_{1}: V_{1} \rightarrow Z$ is a linear mapping with $\left.g_{1}\right|_{V_{0}}=g_{0}$ and satisfying

$$
\begin{aligned}
& \left\|f_{0}(u, y+\lambda t)-g_{1}(y+\lambda t)\right\|_{Z} \\
& \quad=v(\lambda) \cdot\left\|\left(f_{0}\left(u, \lambda^{-1} y+t\right)-g_{0}\left(\lambda^{-1} y\right)\right)-g_{1}(t)\right\|_{Z} \\
& \quad<v(\lambda) \cdot\left(\pi_{u} \circ\left\|\lambda^{-1} y+t\right\|_{Y}\right)=\pi_{u} \circ\left(v(\lambda) \cdot\left\|\lambda^{-1} y+t\right\|_{Y}\right)=\pi_{u} \circ\|y+\lambda t\|_{Y}
\end{aligned}
$$

for all $u \in U_{0}, y \in V_{0}$ and $0 \neq \lambda \in K$. So we have obtained $\left(V_{1}, g_{1}\right) \in \mathfrak{R}$ with $\left(V_{0}, g_{0}\right)<$ $\left(V_{1}, g_{1}\right)$, which is a contradiction.

Consequently, $V_{0}=Y$ holds and $g_{0}: Y \rightarrow Z$ is a linear mapping with

$$
g_{0}(y) \in Z_{\pi_{u} \circ\|y\|_{Y}}\left(f_{0}(u, y)\right) \quad \text { for all } u \in U_{0} \quad \text { and } \quad 0 \neq y \in Y
$$

and $g_{0}(y)=0$ for all $y \in V$. We define

$$
f_{1}(x+\lambda s, y):=f_{0}(x, y)+\lambda g_{0}(y)
$$

for all $x \in U_{0}, \lambda \in K$ and $y \in Y$; hence, $f_{1}: U_{1} \times Y \rightarrow Z$ is a bilinear mapping with $\left.f_{1}\right|_{U_{0}}=f_{0}$ and

$$
f_{1}(x+\lambda s, y)=f_{0}(x, y)+\lambda g_{0}(y)=0
$$

for all $x \in U_{0}, \lambda \in K$ and $y \in V$. Furthermore, for all $x \in U_{0}, 0 \neq \lambda \in K$ and $0 \neq y \in Y$ we have

$$
\begin{aligned}
\left\|f_{1}(x+\lambda s, y)\right\|_{Z} & =v(\lambda) \cdot\left\|f_{0}\left(-\lambda^{-1} x, y\right)-g_{0}(y)\right\|_{Z}<v(\lambda) \cdot\left(\pi_{-\lambda^{-1} x} \circ\|y\|_{Y}\right) \\
& =\left(v(\lambda) \cdot\left\|-\lambda^{-1} x-s\right\|_{X}\right) \circ\|y\|_{Y}=\|x+\lambda s\|_{X} \circ\|y\|_{Y} .
\end{aligned}
$$


Thus, we have $\left(U_{1}, f_{1}\right) \in \mathfrak{M}$ with $\left(U_{0}, f_{0}\right)<\left(U_{1}, f_{1}\right)$ contradicting the maximality of $\left(U_{0}, f_{0}\right)$.

Consequently, $U_{0}=X$ and we can define $F=f_{0}$.

Due to the necessity of modifying the multiplication of a Hahn division algebra of formal power series in an appropriate way, we need the following result. For an additive structure $(G,+)$, we call a mapping $f: G \times G \rightarrow G$ biadditive, if the equations

$$
f\left(g, h^{\prime}+h^{\prime \prime}\right)=f\left(g, h^{\prime}\right)+f\left(g, h^{\prime \prime}\right) \quad \text { and } \quad f\left(g^{\prime}+g^{\prime \prime}, h\right)=f\left(g^{\prime}, h\right)+f\left(g^{\prime \prime}, h\right)
$$

hold for all $g, g^{\prime}, g^{\prime \prime}, h, h^{\prime}, h^{\prime \prime} \in G$.

Theorem 2. Let $(L,+, \cdot)$ be a division algebra endowed with a spherically complete valuation $v: L \rightarrow \Gamma_{0}$, and let $\varphi: L \times L \rightarrow L$ be a biadditive mapping satisfying

- $\varphi(m, 1)=0$ and $\varphi(1, x)=0$ for all $m, x \in L$,

- $v(\varphi(m, x))<v(m) \cdot v(x)$ for all $m, x \in L \backslash\{0\}$.

Then $(L,+, *)$ with $m * x=m \cdot x+\varphi(m, x)$ for all $m, x \in L$ is again a division algebra endowed with the (spherically complete) valuation $v$.

Proof. For all $m, n, x, u \in L$, we have

$$
\begin{aligned}
(m+n) * x & =(m+n) \cdot x+\varphi(m+n, x)=m \cdot x+n \cdot x+\varphi(m, x)+\varphi(n, x) \\
& =(m \cdot x+\varphi(m, x))+(n \cdot x+\varphi(n, x))=m * x+n * x
\end{aligned}
$$

and

$$
1 * x=1 \cdot x+\varphi(1, x)=x
$$

and in an analogous way also

$$
m *(x+u)=m * x+m * u \quad \text { and } \quad m * 1=m
$$

thus, it follows $0 * x=0$ and $m * 0=0$.

For all $m, x \in L \backslash\{0\}$ we have $v(\varphi(m, x))<v(m \cdot x)$ and therefore

$$
v(m * x)=v(m \cdot x+\varphi(m, x))=v(m \cdot x)=v(m) \cdot v(x) .
$$

Finally, let $m, b \in L$ with $m \neq 0$. Since $(L,+, \cdot)$ is a division algebra, there exists $f(x) \in L$ such that

$$
m \cdot f(x)+\varphi(m, x)=b .
$$


For all $x, y \in L$ with $x \neq y$ we have

$$
m \cdot f(x)-m \cdot f(y)=(b-\varphi(m, x))-(b-\varphi(m, y))=\varphi(m, y-x)
$$

and therefore

$$
v(m) \cdot v(f(x)-f(y))=v(\varphi(m, y-x))<v(m) \cdot v(x-y)
$$

hence, $f: L \rightarrow L$ is a strictly contracting mapping of the spherically complete ultrametric space $\left(L, d_{v}, \Gamma_{0}\right)$. By the ultrametric Banach's Fixed Point Theorem [7, Satz 2], there exists exactly one $x_{0} \in L$ such that $f\left(x_{0}\right)=x_{0}$, and we obtain

$$
m * x_{0}=m \cdot x_{0}+\varphi\left(m, x_{0}\right)=b .
$$

Similarly one proves that for all $x, b \in L$ with $x \neq 0$ there exists a unique $m_{0} \in L$ with $m_{0} * x=b$.

In the sequel, we consider a valuation $v: N \rightarrow \Gamma_{0}$ of the division algebra $(N,+, \cdot)$ with value loop $v\left(N^{*}\right)=\Gamma$, and we assume that $N$ has the same characteristic as its residue division algebra $N_{v}=A_{v} / M_{v}$ with $A_{v}=N^{\varepsilon}(0)$ and $M_{v}=N_{\varepsilon}(0)$, i.e., $N$ and $N_{v}$ have the same prime field $P$.

The division algebras $N$ and $N_{v}$ as well as the subgroups $A_{v}$ and $M_{v}$ of $N$ can be regarded as $P$-linear spaces, and the canonical mapping

$$
v: A_{v} \rightarrow N_{v}, \quad x \mapsto x+M_{v}
$$

is a $P$-epimorphism. So there exists a $P$-linear subspace $K$ of $A_{v}$ containing $P$ such that $\left.v\right|_{K}: K \rightarrow N_{v}$ is a $P$-isomorphism. Therefore, $K$ is a system of representatives of the equivalence relation $\equiv_{\varepsilon}^{-}$in $A_{v}$, i.e., for all $x \in A_{v}$ there is a unique $k \in K$ with $v(x-k)<\varepsilon$.

Let $\left(\mathbf{H}, d_{\mathbf{H}}, \Gamma_{0}\right)$ be the Hahn space of formal power series $\mathbf{f}: \Gamma \rightarrow K$ with dually wellordered support $\operatorname{supp}(\mathbf{f})=\{\gamma \in \Gamma \mid \mathbf{f}(\gamma) \neq 0\}$. For all $\gamma \in \Gamma$ we choose elements $u^{\gamma} \in N$ with $v\left(u^{\gamma}\right)=\gamma$ and $u^{\varepsilon}=1$.

For all $d_{v}$-compatible equivalence relations $\sigma \in \equiv(N)$, the equivalence class

$$
V_{\sigma}=[0]_{\sigma}=\{x \in N \mid 0 \sigma x\}
$$

of 0 with respect to $\sigma$ is a $P$-linear subspace of $N$, and we have $V_{\sigma} \subseteq V_{\tau}$ for all $\sigma, \tau \in \equiv(N)$ with $\sigma \subseteq \tau$.

Let $U$ be the $P$-linear subspace of $N$ generated by $\left\{k \cdot u^{\gamma} \mid k \in K\right.$ and $\left.\gamma \in \Gamma\right\}$; for all $\sigma \in \equiv(N)$, the $P$-linear subspace $U_{\sigma}$ of $N$ generated by $\left\{k \cdot u^{\gamma} \mid k \in K\right.$ and $\gamma \in \Gamma$ with $\left.\sigma \subsetneq \equiv_{\gamma}\right\}$ is a $P$-linear complement of $V_{\sigma} \cap U$ in $U$, and $U_{\tau} \subseteq U_{\sigma}$ holds for all $\sigma, \tau \in \equiv(N)$ with $\sigma \subseteq \tau$.

Then, according to Banaschewskis proof of [1, Lemma 4], there exists a family $\left(\zeta\left(V_{\sigma}\right)\right)_{\sigma \in \equiv(N)}$ of $P$-linear subspaces of $N$ with 


$$
\begin{aligned}
& N=V_{\sigma} \oplus \zeta\left(V_{\sigma}\right) \text { for all } \sigma \in \equiv(N), \\
& K \cdot u^{\gamma} \subseteq \zeta\left(V_{\sigma}\right) \quad \text { for all } \gamma \in \Gamma \quad \text { and } \quad \sigma \in \equiv(N) \quad \text { with } \sigma \subseteq \equiv_{\mu}^{-}
\end{aligned}
$$

and

$$
\zeta\left(V_{\tau}\right) \subseteq \zeta\left(V_{\sigma}\right) \text { for all } \sigma, \tau \in \equiv(N) \text { with } \sigma \subseteq \tau
$$

In particular, for all $x \in N$ there exist unique elements $x_{\sigma} \in V_{\sigma}$ and $x_{\sigma}^{\zeta} \in \zeta\left(V_{\sigma}\right)$ with $x=x_{\sigma}+x_{\sigma}^{\zeta}$.

To define a distance-preserving mapping $\theta:\left(N, d_{v}, \Gamma_{0}\right) \rightarrow\left(\mathbf{H}, d_{\mathbf{H}}, \Gamma_{0}\right)$, let $x \in N$ and $\gamma \in \Gamma$. Since $K$ is a system of representatives of $\equiv_{\varepsilon}^{-}$in $A_{v}$, we have $V_{\equiv_{\gamma}}=$ $V_{\equiv-} \oplus K \cdot u^{\gamma}$ and therefore

$$
N=V_{\equiv_{\gamma}} \oplus K \cdot u^{\gamma} \oplus \zeta\left(V_{\equiv_{\gamma}}\right)
$$

thus there is a unique representation

$$
x=x_{\equiv-}+\widehat{x_{\gamma}} \cdot u^{\gamma}+x_{\equiv}^{\zeta}
$$

with $x_{\equiv-\bar{\gamma}} \in V_{\equiv_{\gamma}}, \widehat{x_{\gamma}} \in K$ and $x_{\equiv_{\gamma}}^{\zeta} \in \zeta\left(V_{\equiv_{\gamma}}\right)$. By putting $\theta(x)(\gamma)=\widehat{x_{\gamma}}$ we define a mapping $\theta(x): \Gamma \rightarrow K$ with dually well-ordered support. Indeed, suppose there exists a strictly increasing sequence $\left(\gamma_{n}\right)_{n \in \mathbb{N}}$ in the support of $\theta(x)$. Then $\sigma=\bigcup_{n \in \mathbb{N}} \equiv_{\gamma_{n}}$ is a $d_{v}$-compatible equivalence relation, and we obtain

$$
x-x_{\sigma}=x_{\sigma}^{\zeta} \in \zeta\left(V_{\sigma}\right) \subseteq \zeta\left(V_{\equiv_{\gamma_{n}}}\right),
$$

hence $x_{\equiv_{\gamma_{n}}}=\left(x_{\sigma}\right)_{\equiv_{\gamma_{n}}}$ for all $n \in \mathbb{N}$. Since $x_{\sigma} \in V_{\sigma}$, there is $n_{0} \in \mathbb{N}$ with $x_{\sigma} \in V_{\equiv_{\gamma_{\eta_{0}}}}$, which yields

$$
x_{\equiv_{\gamma_{n_{0}}}}=\left(x_{\sigma}\right)_{\equiv_{\gamma_{n_{0}}}}=x_{\sigma} \in V_{\equiv_{\gamma_{n_{0}}}}
$$

and therefore $\theta(x)\left(\gamma_{n_{0}}\right)=0$, a contradiction to $\gamma_{n_{0}} \in \operatorname{supp}(\theta(x))$.

Consequently, the mapping $\theta: N \rightarrow \mathbf{H}$ is well-defined, and we observe that

$$
d_{v}(x, y)=d_{\mathbf{H}}(\theta(x), \theta(y)) \text { for all } x, y \in N \text {. }
$$

In particular, this implies that $\theta$ is injective. Since we have

$$
\theta\left(k \cdot u^{\gamma}\right)=k t^{\gamma} \quad \text { for all } k \in K \quad \text { and } \quad \gamma \in \Gamma \text {, }
$$

$\left(\theta(N), d_{\mathbf{H}}, \Gamma_{0}\right) \prec\left(\mathbf{H}, d_{\mathbf{H}}, \Gamma_{0}\right)$ is an immediate extension of ultrametric spaces.

Next, we define addition and multiplication on $\mathbf{H}$, such that $\left(\mathbf{H}, v_{\mathbf{H}}, \Gamma_{0}\right)$ becomes a valued division algebra. Hereby, we rely on the construction of a Hahn division algebra presented above. 
First, we have to endow $K$ with a multiplication $\circ$ such that $(K,+, \circ)$ becomes a division algebra. Since $K \subseteq A_{v}=K \oplus M_{v}$ holds, for all $m, x \in K$ we obtain unique elements $m \circ x \in K$ and $r \in M_{v}$ such that

$$
m \cdot x=m \circ x+r
$$

is satisfied. Thus, $(K,+, \circ)$ is a not necessarily associative ring with unit 1 .

Let $m, b \in K$ with $m \neq 0$. Since $N$ is a division algebra, there exists $h \in N$ with

$$
m \cdot h=b,
$$

and since $h \in A_{v}$ there are $x \in K$ and $s \in M_{v}$ with $h=x+s$. Then, by definition of $\circ$, there is $r \in M_{v}$ with $m \cdot x=m \circ x+r$, which yields

$$
K \ni b-m \circ x=m \cdot h-m \cdot x+r=m \cdot s+r \in M_{v}
$$

and therefore

$$
m \circ x=b .
$$

For all $y \in K$ with $m \circ y=b$ it follows $m \circ(x-y)=0$, hence $x=y$. In a similar way we obtain that for all $x, b \in K$ with $x \neq 0$ there is a unique $m \in K$ such that $m \circ x=b$ holds. Thus, $(K,+, \circ)$ is a division algebra.

For all $\alpha, \beta \in \Gamma$ and $m, x \in K$ there exist unique elements $\mu_{\alpha, \beta}(m, x) \in K$ and $r \in V_{\equiv_{\alpha \beta}^{-}}^{-}$ such that

$$
\left(m \cdot u^{\alpha}\right) \cdot\left(x \cdot u^{\beta}\right)=\mu_{\alpha, \beta}(m, x) \cdot u^{\alpha \beta}+r
$$

holds. With the same arguments as above one proves that the family $\left(\mu_{\alpha, \beta}\right)_{\alpha, \beta \in \Gamma}$ of mappings $\mu_{\alpha, \beta}: K \times K \rightarrow K$ is a factor system with respect to $K$ and $\Gamma$. We now endow $\mathbf{H}$ with the corresponding division algebra structure and with the spherically complete valuation $v_{\mathbf{H}}$.

By construction, $\theta$ is $P$-linear, and $v_{\mathbf{H}}(\theta(x))=v(x)$ holds for all $x \in N$. Moreover, $\left(\theta(N), v_{\mathbf{H}}, \Gamma_{0}\right)$ and $\left(\mathbf{H}, v_{\mathbf{H}}, \Gamma_{0}\right)$ can be regarded as valued vector spaces over the (trivially valued) field $P$. The mapping

$$
\varphi: \theta(N) \times \theta(N) \ni(\theta(m), \theta(x)) \mapsto \theta(m \cdot x)-\theta(m) \cdot \theta(x) \in \mathbf{H}
$$

is $P$-bilinear, and by $\theta(1)=\mathbf{1}$ we have $\varphi(\theta(m), \mathbf{1})=\mathbf{0}$ and $\varphi(\mathbf{1}, \theta(x))=\mathbf{0}$ for all $m, x \in N$.

Furthermore, for all $m, x \in N$ with $v(m)=\alpha$ and $v(x)=\beta$ we have unique representations

$$
m=m_{\alpha} \cdot u^{\alpha}+m^{\prime}, \quad x=x_{\beta} \cdot u^{\beta}+x^{\prime} \quad \text { and } \quad m \cdot x=y_{\alpha \beta} \cdot u^{\alpha \beta}+y^{\prime}
$$


with $m_{\alpha}, x_{\beta}, y_{\alpha \beta} \in K$ and $m^{\prime} \in V_{\equiv_{\alpha}^{-}}, x^{\prime} \in V_{\equiv_{\beta}^{-}}, y^{\prime} \in V_{\equiv_{\alpha \beta}^{-}}$. Then

$$
\begin{aligned}
m \cdot x & =\left(m_{\alpha} \cdot u^{\alpha}+m^{\prime}\right) \cdot\left(x_{\beta} \cdot u^{\beta}+x^{\prime}\right) \\
& \in\left(m_{\alpha} \cdot u^{\alpha}\right) \cdot\left(x_{\beta} \cdot u^{\beta}\right)+V_{\equiv_{\alpha \beta}^{-}}=\mu_{\alpha, \beta}\left(m_{\alpha}, x_{\beta}\right) \cdot u^{\alpha \beta}+V_{\equiv_{\alpha \beta}^{-}}
\end{aligned}
$$

yields

$$
y_{\alpha \beta}=\mu_{\alpha, \beta}\left(m_{\alpha}, x_{\beta}\right) .
$$

By

$$
\begin{aligned}
\varphi(\theta(m), \theta(x)) & =\theta(m \cdot x)-\theta(m) \cdot \theta(x) \\
& =\mu_{\alpha, \beta}\left(m_{\alpha}, x_{\beta}\right) t^{\alpha \beta}+\theta\left(y^{\prime}\right)-\left(m_{\alpha} t^{\alpha}+\theta\left(m^{\prime}\right)\right) \cdot\left(x_{\beta} t^{\beta}+\theta\left(x^{\prime}\right)\right)
\end{aligned}
$$

we obtain

$$
v_{\mathbf{H}}(\varphi(\theta(m), \theta(x)))<v_{\mathbf{H}}(\theta(m)) \cdot v_{\mathbf{H}}(\theta(x))
$$

hence, $\varphi$ is strictly contracting.

By Theorem 1, successively applied to both arguments of $\varphi$, there exists a $P$-bilinear and therefore biadditive extension $\Phi$ of $\varphi$ to $\mathbf{H} \times \mathbf{H} \rightarrow \mathbf{H}$ which is strictly contracting and satisfies $\Phi(\mathbf{m}, \mathbf{1})=\mathbf{0}$ and $\Phi(\mathbf{1}, \mathbf{x})=\mathbf{0}$ for all $\mathbf{m}, \mathbf{x} \in \mathbf{H}$.

By Theorem 2, $(\mathbf{H},+, *)$ with $\mathbf{m} * \mathbf{x}=\mathbf{m} \cdot \mathbf{x}+\Phi(\mathbf{m}, \mathbf{x})$ is a division algebra with the spherically complete valuation $v_{\mathbf{H}}$. For all $m, x \in N$ we have

$$
\theta(m \cdot x)=\theta(m) \cdot \theta(x)+\varphi(\theta(m), \theta(x))=\theta(m) * \theta(x)
$$

thus $\theta$ is a value-preserving monomorphism of division algebras from $(N,+, \cdot)$ to $(\mathbf{H},+, *)$. Hence, $\left(\theta(N), v_{\mathbf{H}}, \Gamma_{0}\right) \prec\left(\mathbf{H}, v_{\mathbf{H}}, \Gamma_{0}\right)$ is an immediate extension of valued division algebras.

With these considerations we have shown the following

Theorem 3. Let $\left(N, v, \Gamma_{0}\right)$ be a valued division algebra having the same characteristic as its residue division algebra. Then the following assertions hold:

1. $\left(N, v, \Gamma_{0}\right)$ is maximal, i.e., without any proper immediate extension of valued division algebras, if and only if $\left(N, d_{v}, \Gamma_{0}\right)$ is spherically complete.

2. $\left(N, v, \Gamma_{0}\right)$ possesses a maximal immediate extension, and every maximal immediate extension of $\left(N, v, \Gamma_{0}\right)$ is spherically complete.

This result generalizes [15, Satz 5], which characterizes the valued division algebras admitting an embedding into an appropriate Hahn division algebra of formal power 
series. Finally, [14] gives an example of a division algebra of characteristic 0 with a maximal discrete valuation, i.e., $\Gamma \cong \mathbb{Z}$, which cannot be regarded as a Hahn division algebra.

\section{References}

[1] Banaschewski, B.: Totalgeordnete Moduln. Arch. Math. 7 (1956), 430-440

[2] Brungs, H. H., Törner, G.: Maximal immediate extensions are not necessarily maximally complete. J. Austral. Soc. (Series A) 49 (1990), 196-211

[3] Fleischer, I.: Maximality and ultracompleteness in normed modules. Proc. Amer. Math. Soc. 9 (1958), 151-157

[4] Kalhoff, F.: Uniform Valuations on Planar Ternary Rings. Geom. Dedicata 28 (1988), 337-348

[5] Kaplansky, I.: Maximal Fields with Valuations. Duke Math. J. 9 (1942), 303-321

[6] Kuhlmann, F.-V.: Valuation theory of fields, abelian groups and modules. To appear in: Algebra, Logic and Applications, Gordon and Breach

[7] Prieß-Crampe, S.: Der Banachsche Fixpunktsatz für ultrametrische Räume. Result. Math. 18 (1990), 178-186

[8] Prieß-Crampe, S., Ribenboim, P.: Generalized Ultrametric Spaces I. Abh. Math. Sem. Univ. Hamburg 66 (1996), 55-73

[9] Prieß-Crampe, S., Ribenboim, P.: Homogeneous Ultrametric Spaces. J. of Algebra 186 (1996), 401-435

[10] Prieß-Crampe, S., Ribenboim, P.: Generalized Ultrametric Spaces II. Abh. Math. Sem. Univ. Hamburg 67 (1997), 19-31

[11] Schörner, E.: On Immediate Extension of Ultrametric Spaces. Result. Math. 29 (1996), 361-370

[12] Schörner, E.: Zur Konstruktion von Hahn-Ternärkörpern als Ternärkörper formaler Potenzreihen. Geom. Dedicata 80 (2000), 157-171

[13] Schörner, E.: Hahn-Ternärkörper mit speziellen Faktorsystemen. Geom. Dedicata 80 (2000), 211-230

[14] Schörner, E.: Diskret bewertete Ternärkörper und Hahn-Ternärkörper auf $\mathbb{Z}$. J. of Geometry 71 (2001), 162-181

[15] Schörner, E.: Zur Einbettbarkeit uniform bewerteter Ternärkörper in Hahn-Ternärkörper. Preprint

[16] Zelinsky, D.: Nonassociative valuations. Bull. Amer. Math. Soc. 54 (1948), 175-183

[17] Warner, S.: Topological Fields. North-Holland, Amsterdam, New York, Oxford, Tokyo 1989

Received March 15, 2000; revised April 23, 2001; in final form September 24, 2001

Erwin Schörner, Mathematisches Institut der Universität München, Theresienstraße 39, D-80333 München

schoerner@rz.mathematik.uni-muenchen.de 